\title{
POLÍTICAS PÚBLICAS: DE LA TEORÍA A LA PRÁCTICA
}

\author{
PUBLIC POLICIES: \\ FROM THEORY TO PRACTICE \\ PALABRAS CLAVE: POLÍTICA PÚBLICA * LEGISLACIÓN * \\ CIUDADANÍA * SOCIEDAD \\ KEYWORDS: GOVERNMENT POLICY * LEGISLATION * \\ NATIONALITY * SOCIETY
}

Daniel Camacho Monge

Director

Un rasgo importante de las políticas públicas es que contienen planes complejos y coherentes de mediano o largo plazo, tienen como objetivo a la totalidad de la población o sectores amplios de ella y, por lo menos en la teoría, buscan el bien común. Para su ejecución requieren instrumentos, tales como, la promulgación de normas (leyes, reglamentos, directrices y otros instrumentos jurídicos) recursos financieros, recursos humanos y un grado considerable de legitimidad, entendida como la aceptación y el apoyo por parte de un sector significativo o por la totalidad de la ciudadanía.

Lo anterior no es una definición, sino un intento de señalar los componentes de la política pública para, con el estudio de casos concretos, observar de qué manera se concretan esos componentes en la realidad, ya que el tema central de este número de la Revista de Ciencias Sociales es precisamente la teoría y la práctica de las políticas públicas.

Se justifica el estudio de ese tema porque es bastante frecuente encontrar un desface entre lo formulado en la política pública y su ejecución concreta. En la mayoría de las veces, las resistencias tienen su explicación en el poder, sea material o ideológico.

Una política pública generada desde las más altas instancias de poder formal, como puede ser los poderes ejecutivo y legislativo de un país, puede encontrar resistencias derivadas de la ideología de los operadores de esa política en los niveles concretos de ejecución. Ese es el caso del sesgo de género que, según el artículo de Isabel Gamboa, se observa en los programas de educación primaria costarricense elaborados por el Ministerio de Educación Pública. En ellos se encuentran dos posturas: una liberal que utiliza conceptos como la ciudadanía sexual, expresiones de género, orientación sexual e identidades de género y la otra conservadora que se devela en la forma como concibe el rol de los niños y de las niñas en los juegos $y$ en su comportamiento, así como la ausencia de temas como la violencia sexual o la discriminación salarial. Isabel Gamboa muestra con claridad la causal ideológica de ese desface entre una política igualitaria formulada en diversas normas legales y ampliamente aceptada en la sociedad y su aplicación concreta. 
El poder material se manifiesta en el insuficiente apoyo a las medianas, pequeñas y miniempresas a pesar de las políticas generales que formulan su importancia para el desarrollo, tal como lo analiza Cousin.

Otro desface provocado por el poder material lo describe Rossi, quien encuentra que los planes de estabilización económica y los programas de ajuste estructural, aplicados en su momento en el continente africano, se concretaron de forma tal que favorecieran a los intereses trasnacionales, objetivo muy distante del perseguido por las políticas públicas.

Cuando el ejercicio del poder político tiene una fuerte presencia de los intereses populares, pueden lograrse de mejor manera los objetivos destinados a favorecer a ese sector de la población y eso lo muestran Vives y Naranjo en su artículo que analiza el crecimiento de la región de Riobamba durante los gobiernos de Rafael Correa en El Ecuador.

Un factor que suele distorsionar los objetivos de las políticas públicas es el clientelismo electoral que Rodríguez encuentra responsable de la desnaturalización del funcionamiento de las Asociaciones de Desarrollo Comunal, una institución creada en Costa Rica para lograr la participación de los vecinos en el desarrollo de sus propias comunidades y que ha sido utilizado para fines electorales de ciertos partidos políticos, para mostrarlo el autor estudia la Asociación de Desarrollo Comunal de Zaragoza de Palmares en la provincia de Alajuela de Costa Rica.

La participación comprometida de los propios movimientos populares es un factor determinante para el logro de los objetivos de las políticas públicas, tal como lo muestran Hernández y otros en el artículo en el cual estudian el tema de la educación y la salud en las comunidades indígenas del Chocó en Colombia. Ellos describen el trabajo del grupo Piraguas el cual llevó a cabo un programa para formar promotores en salud quienes se convirtieron en trasmisores de conocimientos y así facilitaron la armonización entre la medicina ancestral y la medicina moderna.

En resumen, la sección central de esta entrega de la Revista de Ciencias Sociales se ha dedicado a estudios de caso en los cuales se hurga en la distancia entre políticas públicas y su aplicación concreta.

Con mayor detalle los artículos de esta sección central se resumen a continuación. Gamboa, realiza un análisis del discurso de los programas de educación primaria costarricense elaborados por el Ministerio de Educación Pública. Se toma como referente teórico a Teun van Dijk y se analizan las variables de la semántica local, el estilo, la argumentación, el relato de anécdotas y las estructuras de la conversación. Se encuentran dos posturas en este estudio: una liberal y la otra conservadora. Por un lado, se encontraron conceptos liberales como la ciudadanía sexual, expresiones de género, orientación sexual e identidades de género. Por otro lado, se evidenció una constante conservadora en indicar el rol en términos de juego y comportamiento de los niños y las niñas, así como la ausencia de temas como la violencia sexual o la discriminación salarial. Se muestra un carácter misógino, en el cual a la mujer se le debe inculcar reglas conservadoras $y$ tener cierto tipo de comportamientos. Como se observa, se presentan dos posturas distintas y contrapuestas dentro de estos 
programas de educación, que infieren en el comportamiento y en la conducta del estudiantado costarricense.

En el área de Historia Económica, Cousin presenta el surgimiento de las pymes en Costa Rica durante el periodo 1950-2018, dividido en dos épocas: modelo de desarrollo de sustitución de importaciones (19481979), enfocado en fortalecer la actividad industrial y el mercado interno, y el modelo de liberación económica (1982-2018), el cual promovía las exportaciones no tradicionales, impulsaba la Inversión Extranjera Directa (IED) y reducía la participación del Estado en la economía costarricense. Esta nueva política económica trajo consigo dificultades para las pymes al eliminar los subsidios y disminuir los créditos a los pequeños agricultores o empresarios, asimismo, afectó negativamente en la competitividad y en la productividad de las pymes, las cuales contaban con poca inversión tecnológica a la que pudieran acceder frente a los grandes avances de las empresas extranjeras.

Rossi realiza un análisis de los Planes de Estabilización Económica $y$ Ajuste Estructural (PAEs) implementados en el continente africano durante la década del 80, como parte de los esfuerzos de las instituciones financieras internacionales como el Fondo Monetario Internacional y el Banco Mundial para impulsar una mayor globalización. Esta decisión se basó en el modelo extrovertido promovido por Badi (1993), el cual se centraba en los principios de economía de desarrollo y liberalismo, estimulando la ayuda externa para fomentar la diversificación de las exportaciones $y$ crear industrias de bienes semifacturados o productos industriales. Sin embargo, este modelo se pensó desde la concepción de economías industriales, sin considerar las características geográficas, económicas, políticas, culturales y sociales de la región. Esto trajo consigo el fracaso de su implementación en este continente, al separar lo político-cultural de lo económico. Asimismo, no se consideró las variables geográficas y demográficas, las cuales son de gran relevancia en términos estructurales, ya que son regiones donde se encontraban grandes problemas como las hambrunas y los conflictos armados.

Desde Ecuador, Vives y Naranjo estudian el contexto ideológico del socialismo en el siglo xxi y la situación económica del cantón Riobamba de este país, durante el gobierno de Rafael Correa en los años 2007-2017. Se evidencia que el proyecto del presidente Correa ha tenido influencias ideológicas importantes: del pensamiento keynesiano, del pensamiento Cepalino sobre la Industrialización y Sustitución de Importaciones; $y$ del Foro de Sao Paolo (motivación de la idea de un nuevo socialismo). Las políticas económicas del gobierno de Rafael Correa tuvieron un impacto sustancial en el desarrollo de las economías locales, las cuales promovían el mejoramiento de las condiciones de vida, una dinámica equilibrada entre sociedad, Estado y mercado, en armonía con la naturaleza; así como el crecimiento del Índice de Desarrollo Humano. En el texto se demuestra como el cantón de Riobamba ha tenido grandes avances durante este periodo, representando el 73,3\% de la economía total de la provincia de Chimborazo, impulsada por las actividades de la Construcción, el Transporte, información $y$ comunicaciones, $y$ la Manufactura. 
En el ámbito de las organizaciones comunales y pueblos rurales, Rodríguez hace un recorrido histórico de la Asociación de Desarrollo Integral en el distrito de Zaragoza en Palmares (Costa Rica) desde la década de 1970. Este tipo de asociaciones velaban por el desarrollo y apoyo de las comunidades, que junto con la mística de los vecinos y vecinas se buscaba impulsar proyectos para la zona, por ejemplo, se realizaban turnos, subastas de ganado, topes, reinados, entre otras, con el propósito de recaudar fondos para la compra de materiales para construcción de parques, aulas, calles, alcantarillas, etc. Es importante el papel que tiene la aparición de DINADECO con un reordenamiento organizativo de los pueblos $y$ los distritos, acompañado por un fuerte involucramiento o manipulación política de los partidos políticos hacia ciertos proyectos e intereses particulares. Además, las élites costarricenses mantenían su injerencia en los pueblos y controlaban en cierta forma las funciones del Estado.

Hernández, Calle, Ospina, Roldán, Álvarez, Jaramillo y Martínez presentan un abordaje desde el ámbito de la educación y la salud para las comunidades indígenas del Chocó en Colombia, a través de un programa para formar promotores en salud que transmitan conocimientos, los apliquen en sus comunidades y establezcan una armonización entre la medicina ancestral y la medicina moderna. El principio fundamental del grupo Piraguas es la promoción de la salud y la prevención de las enfermedades infecciosas presentes en las comunidades indígenas, ya que por su condición cultural y económica son más vulnerables. El grupo Piraguas se ha desplazado a los epicentros de estas comunidades indígenas (a pesar del difícil acesso por sus distancias y barreras físicas como ríos y selvas), con el fin de realizar un aprendizaje bidireccional para que las prácticas y conocimientos se ajusten a las realidad de las comunidades. Como resultado, este proyecto ha formado más de 220 líderes indígenas de este departamento.

En la sección de ARTículos, Haba realiza un ensayo a partir de su opinión sobre el debate entre Peterson-Žižek, denominado "Felicidad: Capitalismo vs. Marxismo" realizado el 19 de abril de 2019. En este caso, se enfoca en las propuestas e ideas de Žižek, las cuales se consideraron simplistas y poco realistas. No se evidenció un debate ni contradicciones entre ambos panelistas. En la primera parte se hace un recuento de la actividad y el abordaje de las temáticas; en la segunda, se hacen algunos aportes metodológicos complementarios; en la tercera, se exponen los resultados, $y$ en la última parte se incluye adicionalmente un aporte desde la intervención del covid-19.

Becerril presenta un aporte desde la teoría política y gobernabilidad, al analizar la Teoría de juegos desde las obras de Hobbes y Maquiavelo. El autor argumenta que tanto la democracia como los regímenes no-democráticos comparten jerarquías como modelos de gobernabilidad. La Teoría de juegos como herramienta de análisis permite determinar la estrategia óptima de acción que los jugadores prefieren, predice resultados de esas interacciones y diseña marcos de interacción enfocados a objetivos específicos. La Teoría de juegos se basa en la teoría de la utilidad, una teoría matemática para representar decisiones. En este estudio se emplea la función de utilidad esperada de Von Neumann con la 
adición de una variante del concepto de tasa de descuento de Selten para refinar equilibrios. Se realizaron varios aportes considerando la región del estado natural y la región de autoimposición, en los temas de proceso, figuras de poder, contrato social, modelo de gobernanza y transiciones.

En la temática de recursos naturales e integración sociocultural, Castañeda, Velasco y Ruiz-Torres realizan una investigación en la comunidad de San Mateo Almomoloa situada en el municipio de Temascaltepec en el Estado de México. A través del método etnográfico se identificaron 5 niveles de integración de orden público en esta comunidad: internacional (interrelación entre la comunidad campesina y las instituciones internacionales que fomentan la conservación del hábitat de la mariposa monarca), nacional (instituciones mexicanas y su normatividad que fomentan el aprovechamiento de los recursos naturales), regional (relación entre las Organizaciones de la Sociedad Civil y las comunidades campesinas), comunitario campesino (gobernanza interna de la población mediante la Ley Agraria, así como, los usos y las costumbres de las comunidades indígenas) y familiar (actividades que las vinculan con el resto de los niveles).

En la colaboración de Martínez-Martínez y Martínez-Carreón se presenta un estudio de percepción subjetiva respecto a la inseguridad y bienestar en cuatro zonas urbanas de México. Según la Encuesta Nacional de Victimización y Percepción sobre Seguridad Pública, el número de víctimas de delito ha ido en un aumento constante en los últimos años, generando sentimientos de impotencia y temor. En este estudio se implementó una entrevista semiestructurada dividida en las dos variables de análisis: inseguridad (subcategorías: percepción de la inseguridad, víctimas del delito, prevención del delito, tipo de delitos en la colonia o localidad) y bienestar subjetivo (subcategorías: bienestar subjetivo positivo, negativo y felicidad). Se evidenció una diferencia en la percepción de seguridad y bienestar según sexo, ya que las mujeres presentan más nerviosismo e intraquilidad, $y$ tienden a preocuparse en mayor medida por sus hijos e hijas; los hombres se preocupan por las pérdidas materiales.

En la sección traducción, Alfaro traduce el ensayo de Henri Lefebvre y Catherine Régulier titulado "El proyecto ritmanalítico", publicado originalmente en francés en Communications, 41, en el año de 1985, pp. 191-199 (L'espace perdu et le temps retrouvé), bajo la dirección de Rémy Lestienne et Edgar Morin. El texto se centra en el concepto de ritmoanálisis como parte de la vida cotidiana desde un enfoque físicobiológico y sociológico. Se pretende brindar un insumo en esta temática tan poco abordada en las ciencias sociales en Latinoamérica.

En la sección de RESEÑAS BIBLIOGRÁFICAS, se cuenta con la colaboración de Montero, quién analiza el libro La Sociología de la Literatura de Gisèle Sapiro. Se analiza la construcción del objeto de estudio entra la Sociología y la Literatura, los marcos teóricos enfocados en la reflexión del texto literario, su creación y la recepción social. Sapiro da un panorama general de la Sociología de la Literatura en el siglo xx, así como demuestra nuevos escenarios para ampliar el objeto de estudio en la actualidad.

Para finalizar, Gomez realiza una reseña del libro La historia Contraataca de Juan Manuel Santana Pérez, historiador venezolano y 
reconocido catedrático de la Facultad de Historia de la Universidad de Las Palmas de Gran Canaria. En este libro se muestra el quehacer de la historiografía contemporánea para dar respuestas a las inquietudes teórico-epistemológicas que han surgido en la investigación en esta área de estudio. El autor parte de una posición hacia el Materialismo Histórico, con el fin de asumir el cambio en las sociedades humanas y establecer una teoría integral de la historia, conservando la relación pasado-presente $y$ futuro relacionando lo objetivo (las condiciones dadas) con lo subjetivo (las personas).

Ciudad Universitaria Rodrigo Facio julio-setiembre, 2020 